\title{
Programmable Locomotion
}

Mechanisms of Nanowires with

Semihard Magnetic Properties

Near a Surface Boundary

\section{Journal Article}

Author(s):

Jang, Bumjin; Hong, Ayoung (D); Alcantara, Carlos; Chatzipirpiridis, George; Martí, Xavi; Pellicer, Eva; Sort, Jordi; Harduf, Yuval; Or, Yizhar; Nelson, Bradley J.; Pané, Salvador

Publication date:

2019-01-23

Permanent link:

https://doi.org/10.3929/ethz-b-000322294

Rights / license:

In Copyright - Non-Commercial Use Permitted

Originally published in:

ACS Applied Materials \& Interfaces 11(3), https://doi.org/10.1021/acsami.8b16907

Funding acknowledgement:

336456 - Magnetoelectric chemonanorobotics for chemical and biomedical applications (EC) 


\title{
Programmable Locomotion Mechanisms of Hard- Magnetic Nanowires Near a Surface Boundary
}

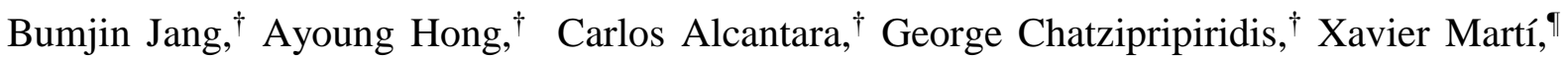 \\ Eva Pellicer, ${ }^{\star}$ Jordi Sort, ${ }^{\star},{ }^{\S}$ Yuval Harduf, ${ }^{\perp}$ Yizhar Or, ${ }^{* \perp}$ Bradley J. Nelson, ${ }^{* \dagger}$ and Salvador \\ Pané*, ${ }^{\dagger}$
}

$\dagger$ Institute of Robotics and Intelligent Systems, ETH Zurich, Zurich, CH-8092, Switzerland

II Institute of Physics, Academy of Sciences of the Czech Republic, Cukrovarnická 10, 162

00 Praha 6, Czech Republic

† Departament de Física, Universitat Autònoma de Barcelona, E-08193 Bellaterra, Spain

$\S$ Institució Catalana de Recerca i Estudis Avançats (ICREA), Pg. Lluís Companys 23, E08010 Barcelona, Spain

$\perp$ Faculty of Mechanical Engineering, Technion Israel Institute of Technology, Haifa, 3200003, Israel

\begin{abstract}
We report on the simplest magnetic nanowire-based surface walker able to change its propulsion mechanism near a surface boundary as a function of the applied rotating magnetic field frequency. The nanowires are made of hard-magnetic CoPt alloy synthesized by means of template-assisted galvanostatic electrodeposition. The hardmagnetic behavior of the nanowires allows for programming their alignment with an applied magnetic field as they can retain their magnetization direction after pre-magnetizing them. By engineering the macroscopic magnetization, the nanowires' speed and locomotion mechanism is set to tumbling, precession, or rolling depending on the frequency of an applied rotating magnetic field. Also, we present a mathematical analysis that predicts the translational speed of the nanowire near the surface, showing very good agreement with experimental results. Interestingly, the maximal speed is obtained at an optimal frequency $(\sim 10 \mathrm{~Hz})$, which is far below the theoretical step-out frequency $(\sim 345 \mathrm{~Hz})$. Finally, vortices
\end{abstract}


are found by tracking polystyrene microbeads, trapped around the CoPt nanowire, when they are propelled by precession and rolling motion.

KEYWORDS: CoPt nanowires, hard magnetism, motion transition, boundary effect, nanopropulsion.

Locomotion adaptability is a fundamental requirement of biomedical micro- and nano-machines, particularly for those that must navigate within complex environments such as the human vasculature. In this context, it is technologically essential to minimize the set of control parameters while maximizing the list of possible manoeuvres. Biology provides fascinating examples of simple control strategies capable of delivering incredible flexibility; by merely changing the direction of just one of its flagellar motors, E. coli can switch between swimming or tumbling via only a single control variable. ${ }^{1}$

In the engineering arena, magnetic fields have become a ubiquitous tool for remotely manipulating nanoobjects. ${ }^{2-9}$ In this article, we describe how to select the speed and the locomotion mechanism of magnetic nanowires near a surface boundary by adjusting only one variable, the frequency of an applied rotating magnetic field. This motion transition using a single input is achieved due to the anisotropy of drag and magnetization of our nanowire, as well as hydrodynamic interaction with the surface. Under the application of a rotating magnetic field, our nanowire propels by tumbling, precessing, or rolling as a function of magnetic field frequency. The underlying principle that governs this behaviour is that magnetic nanowires under a rotating magnetic field change their mode of rotational dynamics from tumbling to precession due to stability transition induced by changing the magnetic field's rotational frequency. ${ }^{10-11}$ The propulsion is generated by drag imbalance along the body caused by the apparent viscosity increase towards the surface by adopting different 
motion mechanisms. ${ }^{12-14}$ The pre-magnetization angle $\theta_{m}$ of the magnetic nanowire, measured from its short axis to the orientation of the applied magnetic field, influences its motion. Previous studies show that tumbling motion occurs near a surface under an external rotating magnetic field where the surface is parallel to $\mathrm{XY}$ plane when a structure is magnetized along its long axis $\left(\theta_{m} \sim 90^{\circ}\right) .^{3,15-17}$ However, when the structure is magnetized along its short axis $\left(\theta_{m} \sim 0^{\circ}\right)$, it rolls on the surface under the same applied rotating magnetic field. ${ }^{18-22}$

Interestingly, a recent analytical model shows that intermediate magnetization orientation scenarios, in which a competition of magnetic torque and hydrodynamics takes place, can lead to tunable locomotion mechanisms. ${ }^{10}$ Motion transformation (tumbling $\rightarrow$ precession $\rightarrow$ nearly rolling) of magnetized nanocylinders in unbounded fluid domain can occur by increasing an applied rotating magnetic frequency, as a consequence of the balance between hydrodynamic and magnetic torques exerted on the body. However, such motion has not been experimentally demonstrated for nano-cylinders due to the difficulty of pre-programing their magnetization orientation during the synthesis. Instead, motion transformation has been primarily demonstrated by helical swimmers. ${ }^{23-25}$ Barbot et al. have recently manufactured helical microswimmers with conical heads that switch between rolling, spintop motion, and corkscrew swimming by adjusting both the direction and the frequency of the rotating magnetic field. ${ }^{26}$

Recent studies on small-scale robotic structures, which are closely related to ours in terms of configuration, are those reported by Zhou et al. and Mair et al. ${ }^{19,27}$ Zhou et al. reported a motion change of a dumbbell-like magnetic microstructure, fabricated by assembling two polystyrene microbeads to the tips of a magnetic nanowire. ${ }^{27}$ They demonstrated that the microstructure "walks" on a solid boundary under a rotating magnetic field. However, the change of motion of the microstructure was due to its weight-imbalance by a slight variation 
in the size of the microbeads. It is difficult to measure a quantitative value for the weightimbalance, which hampers modelling of dynamics of the microstructure. Additionally, this system is much more complicated than ours in terms of manufacturing, as the assembly of the beads to the nanowire tips is random and requires several attempts. In addition, Mair et al. fabricated a nanowire (diameter: $280 \mathrm{~nm}$, length: $5.5 \mu \mathrm{m}$ ) that consists of a thin Ni disk (a thickness of $\sim 80 \mathrm{~nm}$ ) between two long Au segments. This study shows that the nanowire walks on a solid boundary by precession motion under kilohertz frequency of a rotating magnetic field. ${ }^{19}$ Their study assumed that the precession angle is not affected by the frequency of magnetic field. However, any magnetic structure in an elongated shape with a certain magnetization angle $\left(\theta_{m}\right)$ undergoes changes in its precession angle as frequency increases. Also, they did not study the influence on transitions between motion modes with varying the actuation frequency. More importantly, despite the similarity in the configuration of the two works, a proper understanding of the dependence of speed on different configurations, by considering a certain type of rod-surface interaction were not addressed.

Here, for the first time we employ a single hard-magnetic nanowire to demonstrate a complex mechanism of locomotion under an applied magnetic field. The pre-magnetization nature of our samples allows for different motion mechanisms of nanowires according to the rotating frequency of the magnetic field. Our theoretical modelling captures the nanowire's dynamics on a surface boundary by varying magnetic field frequency. The simulated translational velocity on a surface boundary agrees with our experimental data in the same order of magnitude and in the trend of frequency dependence.

\section{RESULTS AND DISCUSSION}

Fabrication and characterization of CoPt hard-magnetic nanowires. Figure 1a shows the synthesis procedure of $\mathrm{CoPt}$ hard-magnetic nanowires up to the pre-magnetization 
method. CoPt nanowires were electrochemically grown in an anodic aluminum oxide (AAO) membrane at a current density of $-30 \mathrm{~mA} \mathrm{~cm}^{-2}$. The CoPt nanowires were subsequently released by etching the membrane in $\mathrm{NaOH}$ solution, followed by cleaning with DI water to remove residuals. A suspension of highly diluted CoPt nanowires was dispersed on a precleaned glass substrate and dried in ambient. Subsequently, an epoxy resin was applied on the deposited side of the substrate and dried in ambient. Next, the CoPt nanowires were premagnetized by saturating magnetic field $(20 \mathrm{kOe})$ seemingly in the direction of the perpendicular to nanowire's axis. The morphology and composition of CoPt nanowires were investigated using scanning electron microscopy (SEM) and energy-dispersive X-ray spectroscopy (EDX). Figure 1b (i) shows the one-dimensional feature of nanowires with a diameter of $200 \mathrm{~nm}$ and a length of $5 \mu \mathrm{m}$. Detected signals of Co (Figure 1b (ii)) and Pt (Figure $1 \mathrm{~b}$ (iii)) confirm the chemical composition of the nanowires, containing only Co (71 \pm 1 wt.\%) and Pt (29 \pm 4 wt.\%). The crystallographic structure of the nanowires was studied by X-ray diffraction (XRD). Figure S1 in the supplementary information (SI) shows the XRD patterns of the nanowires grown at $\mathrm{j}=-30 \mathrm{~mA} \mathrm{~cm}^{-2}$, together with the corresponding Rietveld refinement using the MAUD software. ${ }^{28}$ Nanowires obtained at these deposition conditions consist of two solid solutions: Co-rich hexagonal-close-packed (HCP) and Pt-rich face-centered cubic (FCC) solutions. The values of cell parameters, average crystallite size (coherent diffracting length), microstrains and stacking fault probability obtained from the Rietveld refinements are shown in Table S1. The values of cell parameters $(a$ and $c)$ in the HPC solid solution differ from those of pure Co $(a=0.2507 \mathrm{~nm} ; c=0.4069 \mathrm{~nm})$. Similarly, the values of $a$ in the FCC phases are slightly different from that for pure Pt $(a=0.3924 \mathrm{~nm})$. This confirms the occurrence of the two solid solutions. The magnetic response of the CoPt nanowires was characterized using a VSM from the nanowires still embedded in the AAO template (Figure 1c). The magnetic hysteresis loop of CoPt nanowires obtained with an 
applied field perpendicular to the nanowire's long shows a coercivity of $4 \mathrm{kOe}$ and a squareness ratio, $M_{r} / M_{s}$ (where $M_{r}$ and $M_{s}$ are the remanent and saturation magnetization, respectively) of 0.81 , indicating that our CoPt nanowires are magnetically hard. The hardmagnetic properties of CoPt nanowires allow pre-programming their magnetization direction.

Table S2 lists the coercive field and squareness ratio value for $\mathrm{CoPt}$ nanowires.

(a)

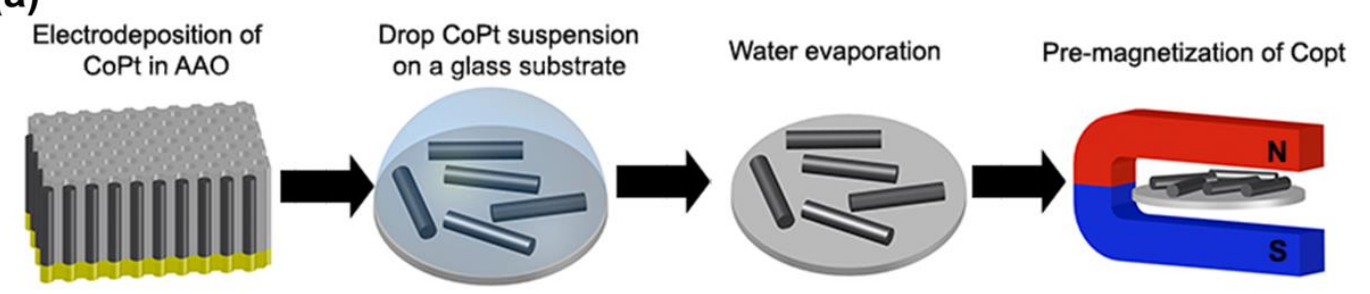

(b)
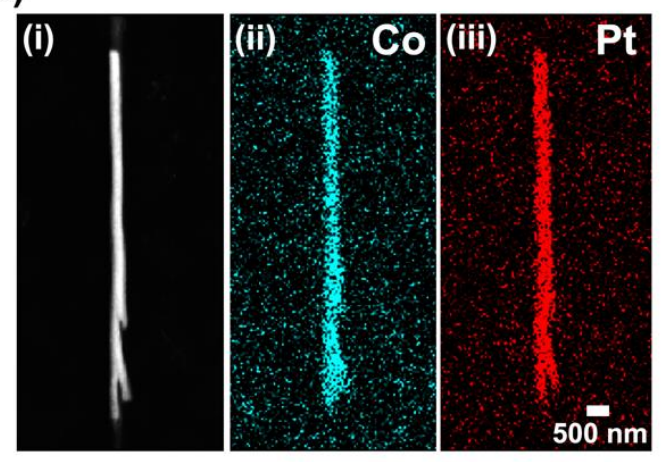

(c)

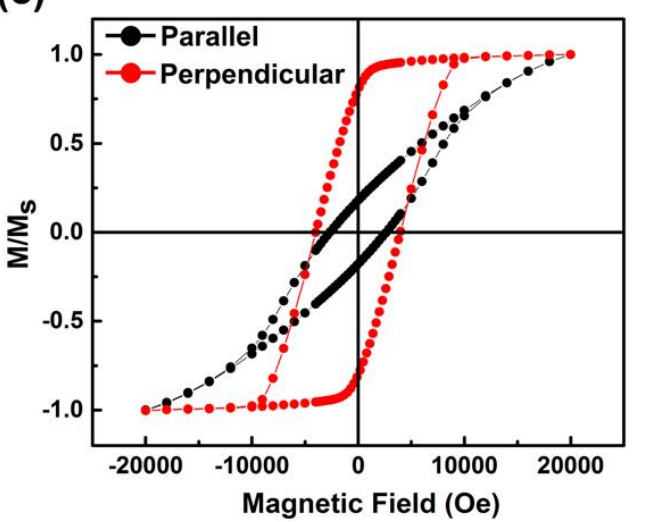

Figure 1. (a) Schematic of the synthesis procedure of CoPt nanowires up to the pre-magnetization process. (b): (i) SEM image and (ii) and (iii) EDX mapping of a single CoPt nanowire. (c) A hysteresis magnetic loop of the pre-magnetized CoPt nanowires in the direction parallel and perpendicular to the long axis of the nanowires.

Magnetization orientation of CoPt hard-magnetic nanowires. Magnetic manipulation of nanowires was experimentally performed on an electromagnetic system, consisting of four pairs of electromagnets as described in Kummer et al. ${ }^{29}$ First, the magnetization orientation $\theta_{m}$ of the pre-magnetized CoPt nanowires was investigated by measuring the angle between the applied magnetic field and the resulting direction of nanowire's short axis. Figure 2a indicates that $\theta_{m}$ is $\sim 25^{\circ}$. This value differs from the targeted pre-magnetization direction (around $0^{\circ}$ ) probably due to the competition between shape and crystalline magnetic 
anisotropies. In contrast, CoNi nanowires (a control sample with the same dimensions) show $\theta_{m} \sim 90^{\circ}$, which is universally observed for soft-magnetic materials as magnetic shape anisotropy dominates over other factors (Figure $2 b) .{ }^{30-31}$

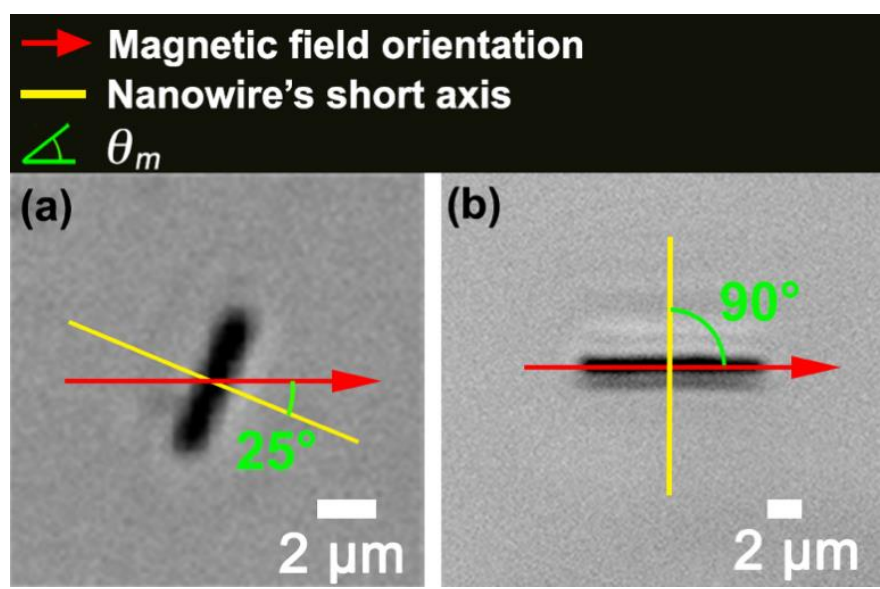

Figure 2. Resulting magnetization angle $\left(\theta_{m}\right)$ of hard- and soft magnetic nanowires. (a) CoPt (b) CoNi.

\section{The spinning motion of CoPt hard-magnetic nanowires under an applied magnetic}

field rotating around the $\mathbf{Z}$-axis. Nanowire motion was studied under an applied rotating magnetic field parallel to the XY-plane of the surface. The magnetic field strength and rotating frequency applied were $8 \mathrm{mT}$ and $10 \mathrm{~Hz}$, respectively. The control sample (CoNi nanowire) with $\theta_{m}=90^{\circ}$ shows a rotational motion within the XY-plane (Figure 3a and c, and movie S1). This result is valid for nanowires composed of soft-magnetic materials. ${ }^{32-33}$ In contrast, the CoPt nanowire with $\theta_{m} \sim 25^{\circ}$ shows out-of-plane motion, rotating about its long axis (Figure $3 \mathrm{~b}$ and $\mathrm{d}$, and movie $\mathrm{S} 2$ ). Such spin-top motion might be useful in certain biological applications, such as drilling a tiny hole in the cell membrane for targeted drug delivery and sensing. 

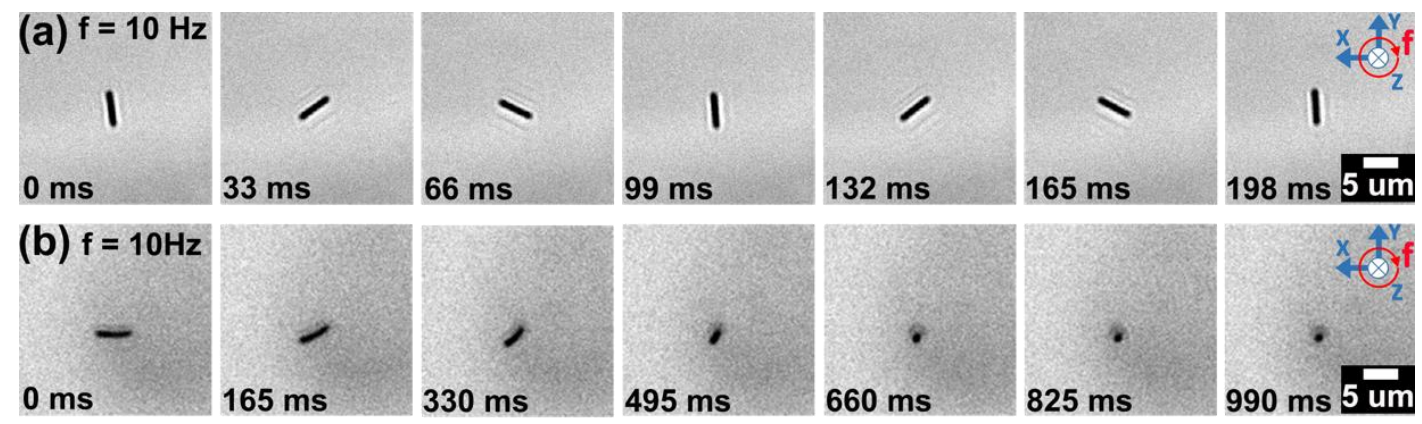

(c) Rotation around the short axis

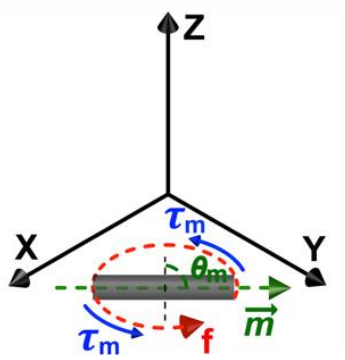

(d) Rotation around the long axis

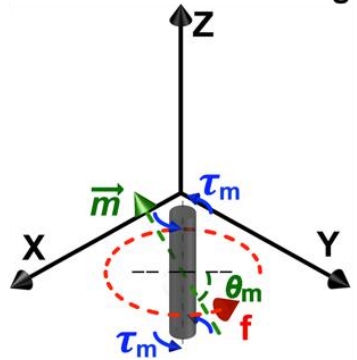

Figure 3. The difference in motion between CoNi (control sample) and CoPt nanowire under an applied magnetic field rotating in the XY-plane. Image sequences of (a) a CoNi nanowire and (b) a CoPt nanowire. Motions of CoNi and CoPt nanowires are depicted in (c) and (d), respectively.

The propulsion of CoPt hard-magnetic nanowires and their motion transition under an applied magnetic field rotating around $\mathbf{X}$-axis. One of the strategies for structures to create locomotion in Stokes flow is to use drag imbalance caused by an increase of the apparent viscosity towards the boundary. ${ }^{12}$ In order to translate CoPt nanowires along the surface in the XY-plane, CoPt nanowires were exposed to an external YZ-planar rotating magnetic field. We observed a motion transition as the rotating frequency increases from $1 \mathrm{~Hz}$ to $15 \mathrm{~Hz}$ at a field strength of $15 \mathrm{mT}$. The precession angle $\psi$ is determined by measuring the maximum angle of the nanowire with respect to $\mathrm{x}$-axis from top view image. When the rotating frequency is small enough $(\sim 1 \mathrm{~Hz})$, CoPt nanowires exhibit tumbling motion in the YZ-plane $\left(\psi \sim 90^{\circ}\right)$, confirmed by a decrease in the projected length of the nanowire (Figure 4a). By increasing the frequency towards $5 \mathrm{~Hz}$, the nanowire starts to move out of the YZplane and shows a precession motion $\left(\psi \sim 48.07^{\circ}\right)$, as can be seen in Figure $4 \mathrm{~b}$. A further increase in frequency leads to a nearly rolling motion, similar to a magnetic sphere demonstrated by Driscoll et al.. ${ }^{34}$ It is determined by a considerably lower precession angle 
$\left(\psi \sim 6.19^{\circ}\right)$, as shown in Figure 4c. The schematic of the different locomotion modes (tumbling, precession, and nearly rolling) can be visualized in movie S3. As the frequency of the rotating field increases, the nanowire changes its motion to experience a lower drag force, resulting in a decrease in precession angle. According to our experimental results, the critical frequency $\left(f_{c}\right)$ under which the motion starts to switch from pure tumbling $\left(\psi=90^{\circ}\right.$ to precession $\left(\psi<90^{\circ}\right)$ is aroud $3 \mathrm{~Hz}$.

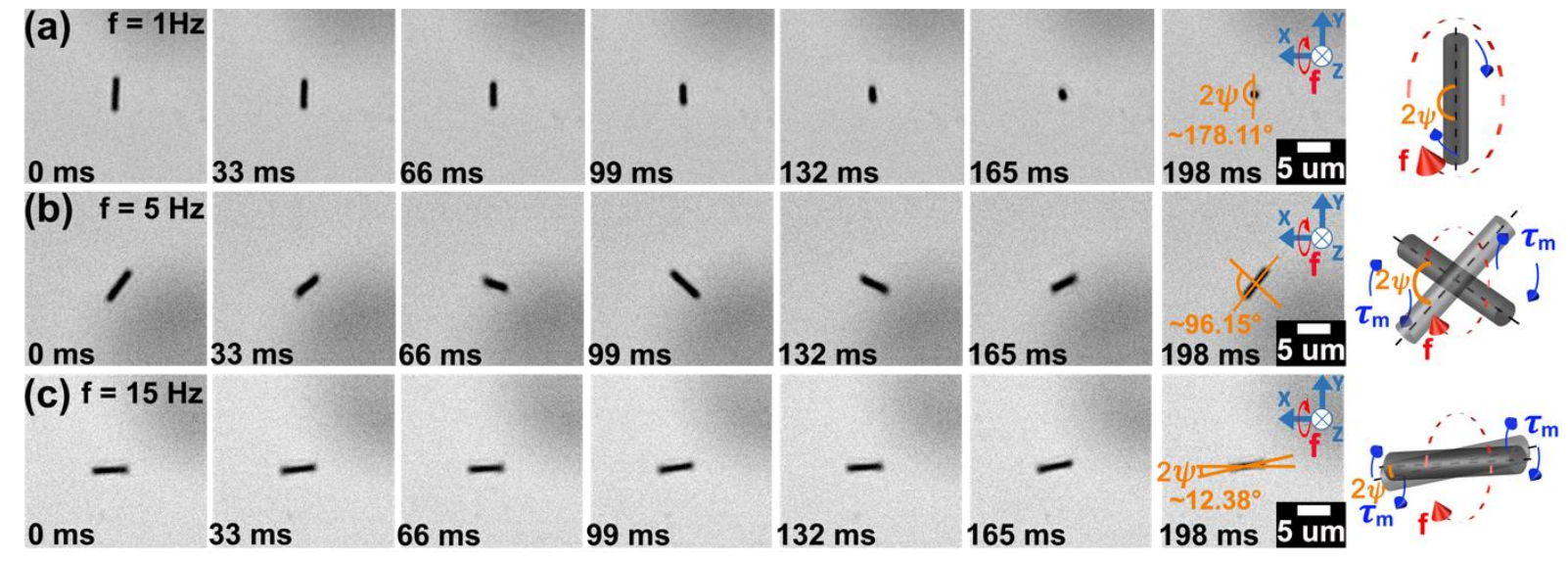

Figure 4. Different locomotion modes of a single CoPt nanowire as function of the frequency of a rotating magnetic field $(15 \mathrm{mT})$ in the YZ-plane. Image sequences for the (a) tumbling motion at a frequency of $1 \mathrm{~Hz}$ (b) precession motion at a frequency of $5 \mathrm{~Hz}$, and (c) rolling motion at a frequency of $15 \mathrm{~Hz}$. The total time lapse is $198 \mathrm{~ms}$. Each locomotion mode is depicted in the right panels.

Theoretical modeling of the transversally magnetized nanowire translating near a wall. A magnetic nanowire with a certain misalignment angle $\theta_{m}$ shows different motion configurations under a rotating magnetic field depending on its applied frequency $f_{B}$. The nanowire shows tumbling motion at low frequencies and enters into a precession regime as the frequency increases. Until the step-out frequency, the nanowire synchronously rotates with the magnetic field. According to Ghosh et al. ${ }^{10}$ who studied the rotational motion of a magnetized nanowire in an unbounded fluid, the transition from tumbling to precession motion occurs at the critical frequency,

$f_{c}=m B \sin \theta_{m} / \gamma_{s}$ 
and the phase-locked precession is maintained until the step-out frequency,

$f_{\text {s.o }}=m B \sqrt{\left(\frac{\sin \theta_{m}}{\gamma_{s}}\right)^{2}+\left(\frac{\cos \theta_{m}}{\gamma_{l}}\right)^{2}}$

where $m$ is the magnitude of magnetic moment and $B$ is the strength of the applied external magnetic field. For a cylindrical object with a half body length $l$ and a radius $R$, the drag coefficient of the nanowire around its short axis and long axis are defined as

$\gamma_{S}=\frac{8 \pi \eta l^{3}}{3\left(\ln \left(\frac{l}{R}\right)+\left(-0.662+\frac{0.917 R}{l}-\frac{0.05 R^{2}}{l^{2}}\right)\right)}$ and $\gamma_{l}=\frac{8 \pi \eta l^{3}}{0.96}\left(\frac{l^{2}}{R^{2}}+0.677 \frac{l}{R}-0.183\right)$, respectively.

The precession angle ${ }^{10}$ of a nanowire in unbounded fluid is defined as

$\psi=\left\{\begin{array}{cl}90^{\circ} & f \leq f_{c} \\ \operatorname{asin}\left(\frac{m B \sin \theta_{m}}{\gamma_{s} f_{B}}\right) & f>f_{c}\end{array}\right.$.

As the applied magnetic field rotates around the $\mathrm{X}$-axis, the body also rotates in a constant angular velocity with $\Omega_{X}=2 \pi f_{B}$. The direction of the unit vector along the body's long axis is obtained by

$\widehat{\boldsymbol{u}}(t)=\cos \psi \hat{\mathbf{x}}-\sin \psi \sin \Omega_{X} t \hat{\mathbf{y}}-\sin \psi \cos \Omega_{X} t \hat{\mathbf{z}}$

in a precession regime where $f>f_{c}$. This is the nanowire's orientation rotating in a symmetric double cone, centered about the X-axis (see Figure 5). When the body tumbles at low frequencies $f<f_{c}$, the body rotates within the YZ-plane, as it can be seen by substituting $\psi=90^{\circ}$ in equation (4).

We have assumed that diffusion, electrostatic, and van der Waals forces are negligible in comparison to the magnetic torque, the hydrodynamic and gravitational forces. Among those considered forces, the magnetic torque dictates the orientation of the body and therefore the nanowire keeps the same rotational kinematics of a double cone as in equation (4). 
To study the propulsion speed of a nanowire near the boundary as a function of the applied magnetic field, we adopted Yang's model. ${ }^{35}$ The motion of a slender body in Stokes flow is considered and therefore, the inertial forces are negligible compared to the viscous forces. The model is constructed based on an asymptotic expansion for small $R / d$, where $d$ is the distance from the center of the body to the surface of the boundary. The projected body frame $\left(\hat{\boldsymbol{e}}_{1}, \hat{\boldsymbol{e}}_{2}, \hat{\boldsymbol{e}}_{3}\right)$ is used to describe the body configuration from Yang's model. In an arbitrary configuration, the body's long axis $\widehat{\boldsymbol{u}}$ makes an angle $\theta$ with respect to the XY-plane, and the unit vector $\hat{\boldsymbol{e}}_{\mathbf{1}}$ is directed along the projection of $\widehat{\boldsymbol{u}}$ on the XY-plane. The rotation angle between $\hat{\boldsymbol{e}}_{\mathbf{1}}$ and the $\mathrm{X}$-axis is denoted as $\phi$. By using equation (4), these two angles are obtained as

$\theta(t)=\operatorname{asin}\left(\sin \psi \cos \Omega_{X} t\right)$

$\phi(t)=\operatorname{asin}\left(\sin \psi \sin \Omega_{X} t / \cos \theta\right)$

The distance from the body tip to the surface is defined as $d_{t}=d-l \sin \theta$.

The governing equation for the dynamics of a nanowire in the world frame is as follows:

$\boldsymbol{F}_{\text {ext }}+\boldsymbol{F}_{\text {drag }}=0$

where $\boldsymbol{F}_{\text {ext }}$ is the external forces and $\boldsymbol{F}_{\text {drag }}$ is the hydrodynamic drag force applied on the nanowire. The translational velocity $(\boldsymbol{U})$ is generated by drag imbalance along the body with a rotational velocity $(\boldsymbol{\Omega})$ near the wall. ${ }^{12}$ The external forces, including the gravitational and buoyant force, are exerted on the body in the z-axis, so that $\boldsymbol{F}_{\text {ext }}=\left[\begin{array}{lll}0 & 0 & \Delta \rho g V\end{array}\right]^{T}$, where $\Delta \rho$ is the density difference between the object and the liquid. The hydrodynamic force applied on the body near the wall is described as,

$\boldsymbol{F}_{\text {drag }}^{e}=\boldsymbol{K}_{T} \boldsymbol{U}^{e}+\boldsymbol{K}_{C}^{T} \boldsymbol{\Omega}^{e}$

where $\boldsymbol{K}_{T}=\left[\begin{array}{ccc}K_{T}^{11} & 0 & K_{T}^{13} \\ 0 & K_{T}^{22} & 0 \\ K_{T}^{31} & 0 & K_{T}^{33}\end{array}\right], \boldsymbol{K}_{C}=\left[\begin{array}{ccc}0 & K_{C}^{12} & 0 \\ K_{C}^{21} & 0 & K_{C}^{23} \\ 0 & K_{C}^{32} & 0\end{array}\right]$. 
Here, $\boldsymbol{K}_{T}$ is the translational resistance tensor and $\boldsymbol{K}_{C}$ is the coupling tensor describing the interaction between translational and rotational motion. Each element of these resistance tensors describes a relation between the hydrodynamic force and its translational velocity or its angular velocity. For example, $K_{T}^{11}$ or $K_{C}^{21}$ indicate the hydrodynamic resistance in the $\hat{\boldsymbol{e}}_{1}$-axis when it moves along $\hat{\boldsymbol{e}}_{\mathbf{1}}$ direction or rotates around $\hat{\boldsymbol{e}}_{\mathbf{2}}$-axis, respectively. The tensor coefficients are functions of the body tilt angle $\theta$, the distance of the body center from the surface $d$, the dynamic viscosity of a liquid $\eta$, and the body slenderness $\epsilon=\ln \left(\frac{2 l}{R}\right)^{-1}$. These coefficients are obtained by integrating the line density of Stokeslet distribution along the body and the detailed formula can be found in Supporting information S1. The translational and angular velocities ( $\boldsymbol{U}^{e}$ and $\boldsymbol{\Omega}^{e}$ ) in (8) are expressed in the projected body frame $\left(\hat{\boldsymbol{e}}_{1}, \hat{\boldsymbol{e}}_{2}, \hat{\boldsymbol{e}}_{3}\right)$. When the nanowire rotates with a given frequency around the X-axis (or angular velocity $\Omega_{X}$ ) near the wall, the instantaneous translational velocity in a fixed frame is obtained from equation (7) and (8) as:

$\boldsymbol{U}=\boldsymbol{Q}^{T} \boldsymbol{K}_{T}^{-1}\left(-\boldsymbol{Q} \boldsymbol{F}_{\text {ext }}-\boldsymbol{K}_{C}^{T} \boldsymbol{Q} \boldsymbol{\Omega}\right)$

where $\boldsymbol{Q}=\left[\begin{array}{ccc}\cos \phi & \sin \phi & 0 \\ -\sin \phi & \cos \phi & 0 \\ 0 & 0 & 1\end{array}\right]$ is the rotation matrix from fixed frame to the projected body frame. Finally, the translational velocity is averaged as $\frac{1}{T} \int \boldsymbol{U} d t$ using (9) with $\boldsymbol{K}_{T}, \boldsymbol{K}_{C}$, and $\boldsymbol{Q}$ updated according to the angles $(\theta$ and $\phi)$ and the distance obtained by the equation $\dot{d}=-U_{Z}$ 

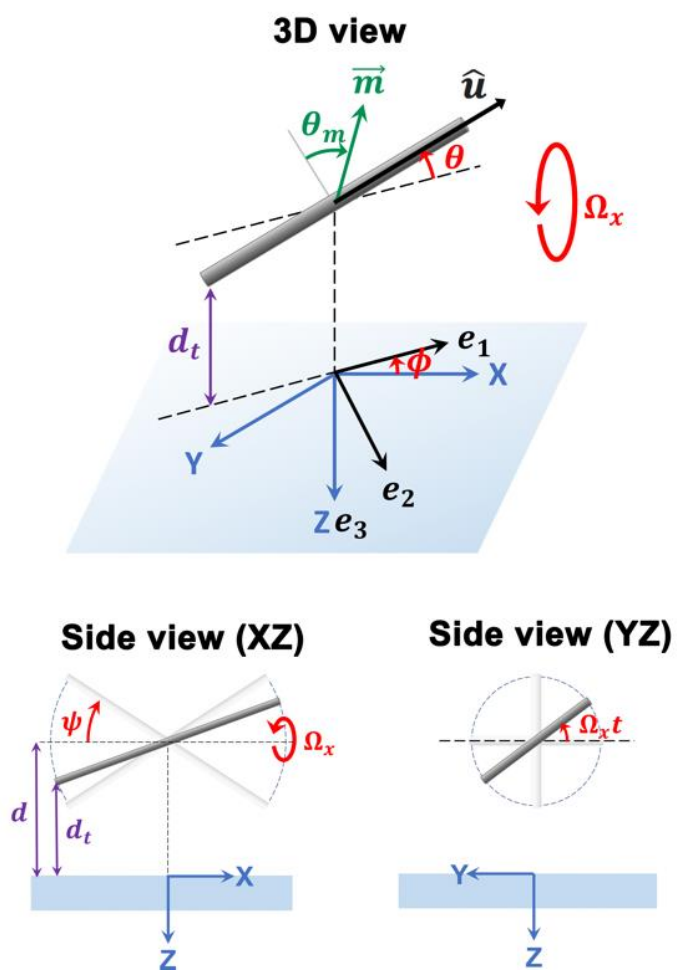

Figure 5. A nanowire with a magnetic moment with magnitude $m$ and misalignment angle $\theta_{m}$ in an arbitrary configuration. The vector $\hat{\boldsymbol{e}}_{\mathbf{1}}$ is defined by projecting $\widehat{\boldsymbol{u}}$ onto the surface. The direction of $\hat{\boldsymbol{e}}_{\mathbf{3}}$ is normal to the wall and $\hat{\boldsymbol{e}}_{2}$ is constructed following the right-hand rule.

Validation of the theoretical models with experimental results. We employed the analytical model introduced above, substituting the physical values of $\theta_{m}=25^{\circ}, B=15 \mathrm{mT}$, and $\eta=8.82 \times 10^{-3} \mathrm{~Pa} \cdot \mathrm{s}(60 \%$ glycerol $)$ at different magnitudes of magnetic moment $(\mathrm{m})$. In Figure $6, \cos \psi$ as a function of frequency from experiments are plotted and overlaid with analytical solutions from equation (3) for various magnitudes of magnetic moment. The precession angle $\psi$ is estimated from experiments by measuring the maximal angle between the nanowire's projected axis $\hat{e}_{1}$ and the $\mathrm{X}$-axis from the $\mathrm{XY}$-view of the camera as seen in Figure 4. The nanowires used in our experiments have a magnetic moment around 1000 emu/g (VSM data in Figure S2), agreeing with the expected analytical solutions. In this case, the critical frequency of our structure is about $f_{c}=3.68 \mathrm{~Hz}$ by equation (1). Also, the 
calculated step-out frequency by equation (2) is $356 \mathrm{~Hz}$, which indicates that phase-locked precession is maintained within the frequency range $(0 \sim 25 \mathrm{~Hz})$ used for our experiments.

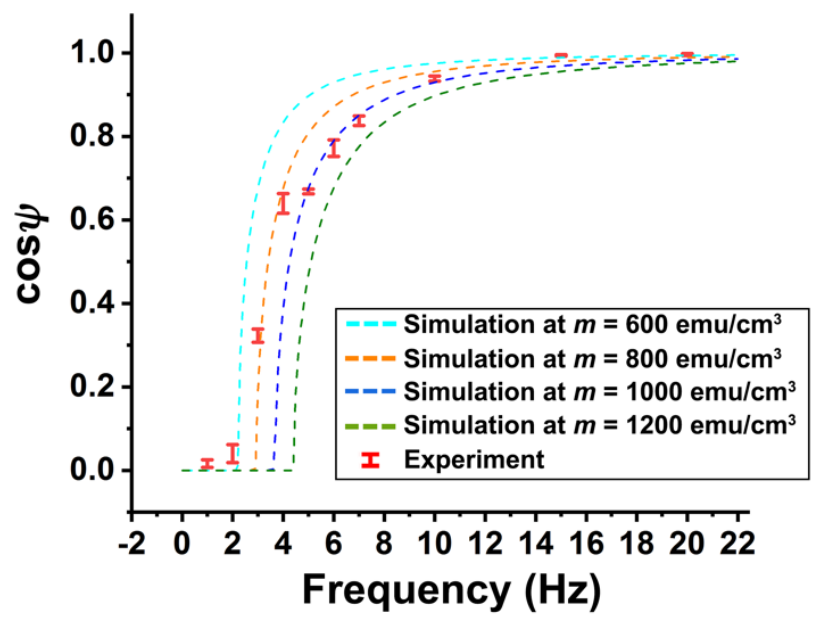

Figure 6. Simulation results and experimental data of precession angles $\psi$ as a function of the frequency of the external rotating magnetic field.

We simulated the total drag forces and translational velocities for three different rotating frequencies $(1,5$, and $15 \mathrm{~Hz}$ ) using equation (8). Figure $7 \mathrm{a}$ shows the $\mathrm{X}, \mathrm{Y}$, and $\mathrm{Z}$ components of resultant drag forces and velocities as a function of the simulation time $(t)$ divided by the rotation period $(T)$. In Figure $7 b$, the trajectory of the nanowire's center in the XY-plane are obtained from integrating velocities. Note that, according to our calculation, the external force (gravitational and buoyant forces, $\Delta \rho g V \sim 5.26 \times 10^{-14} \mathrm{~N}$ ) is one order of magnitude smaller than hydrodynamic drag force in the Z-axis, which drives us to neglect the gravity effect for the following simulation. Nevertheless, the external force effect is discussed in Supporting information S2.

The drag force and velocity on the $\mathrm{X}$-axis are negligible when the nanowire tumbles in the YZ-plane under $1 \mathrm{~Hz}$ of rotating frequency of the external magnetic field. In contrast, drag forces and velocities on the $\mathrm{X}$-axis are observed for precession motion at 5 and $15 \mathrm{~Hz}$ due to 
its $3 \mathrm{D}$ rotational characteristics, presenting a maximum value where $\theta(t)$ and $\phi(t)$ are zero and maximum, respectively. Recall 1 and $3 \mathrm{~Hz}$ are categorized in a tumbling regime whereas 5, 10, 15 and $50 \mathrm{~Hz}$ are in a precession regime with respect to the critical frequency. Although a close look at the trajectories in Figure $7 \mathrm{~b}$ shows a clear X-directional motion toward the direction of its lower half-body oriented, the net motion in the X-axis over one period of rotation is zero as the mean velocities are zero.

In the $\mathrm{Y}$-axis, the nanowire near the wall experiences drag forces, regardless of its locomotion modes (or the rotating frequency). The maximal drag forces and velocities appear when $\theta(t)$ and $\phi(t)$ are maximum and zero, respectively. The vertical offset (or non-zero average values) of Y-directional drag forces and velocities, mainly contributes to the net motion of the body as one can see in Figure $7 b$.

In the Z-axis, as the angle $\theta$ changes from zero to its maximum, the lower half-body of the nanowire pushes the fluids close to the wall yielding a drag force away from the wall. In contrast, as the $\theta$ changes from its maximum to zero, the drag force is observed toward the wall. The drag forces and velocities are consequently oscillating around zero over a period of $\theta$ (or $\mathrm{t} / \mathrm{T})$, presenting a periodic $d_{t}$ profile over the cycles and zero average values. Drag profiles in the $\mathrm{YZ}$ plane in Figure 7c show a higher drag force for the segment close to the wall. In other words, as $\theta$ decreases from its maximum to zero the drag profile becomes symmetrical along the body. 
(a) (i) Drag forces

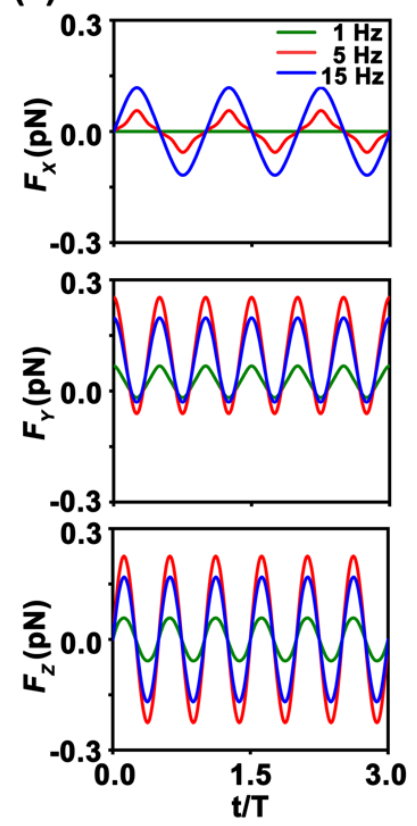

(c)

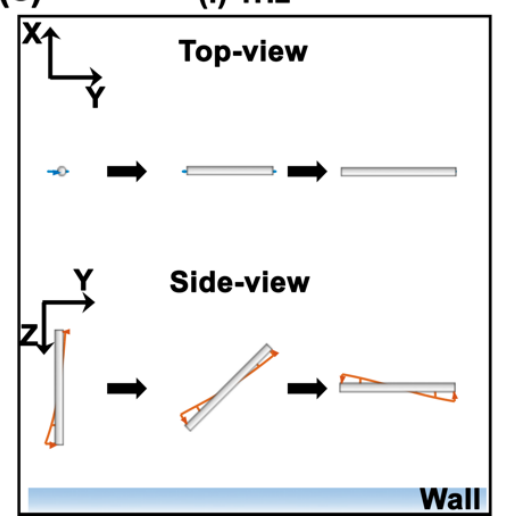

(ii) Velocities

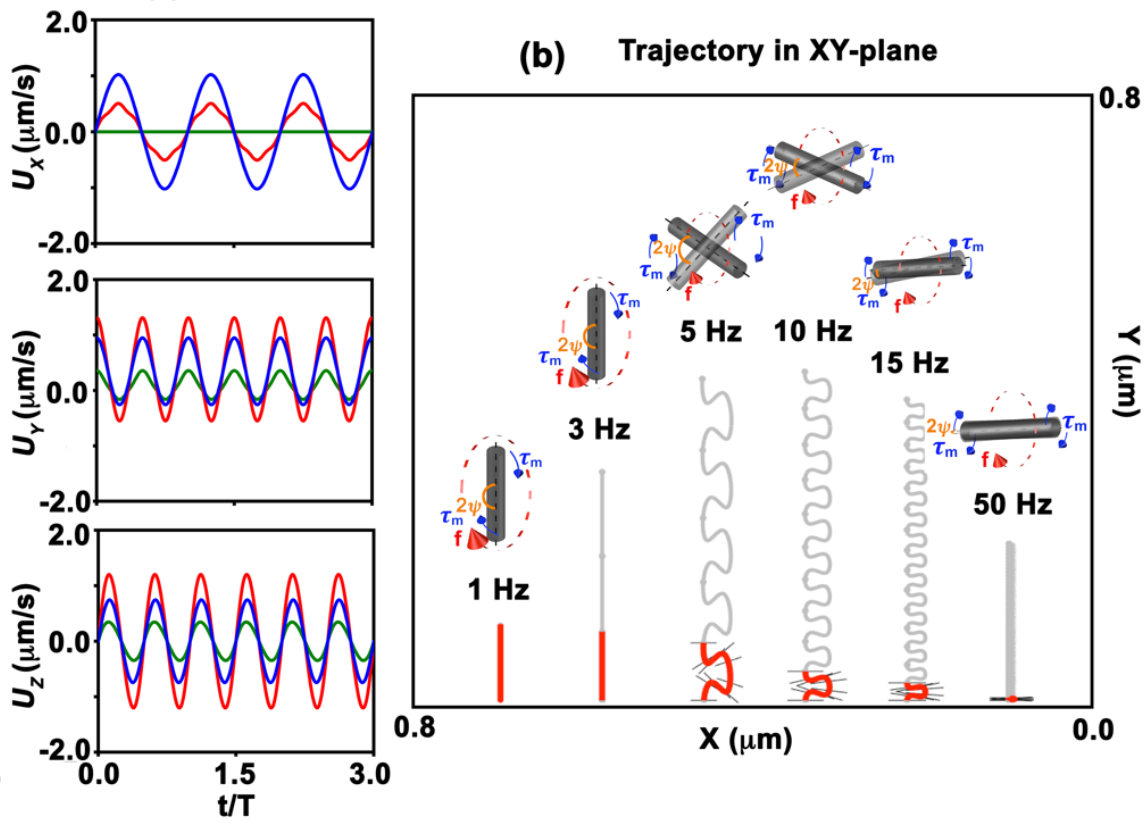

(ii) $5 \mathrm{~Hz}$

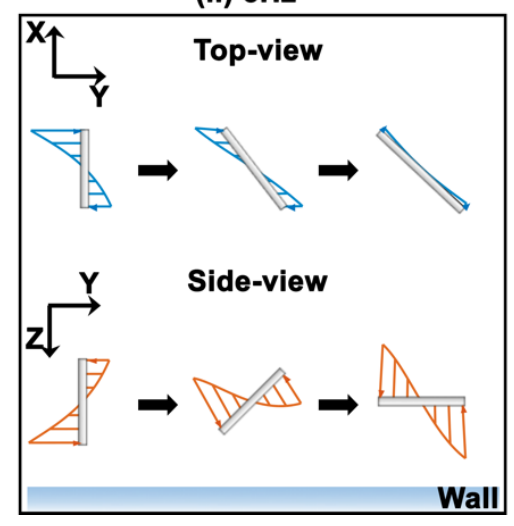

(iii) $15 \mathrm{~Hz}$

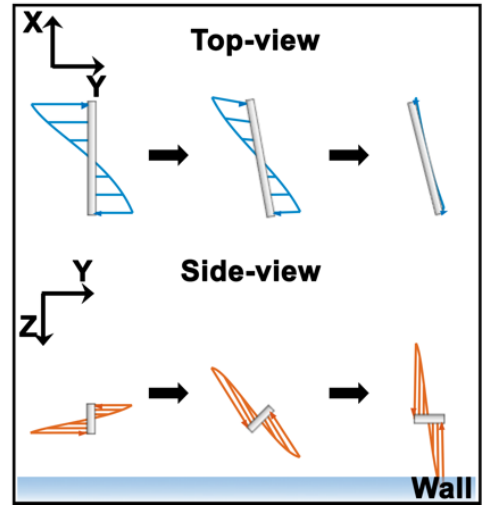

Figure 7. (a) (i) Components of hydrodynamic forces and (ii) velocities of the nanowire when it rotates around the $\mathrm{X}$-axis near the wall. The simulation was conducted up to three periods. Green lines: $1 \mathrm{~Hz}$, red lines:5Hz, and blue lines:15Hz. (b) Simulated XY trajectories of nanowires rotating near the wall at different frequencies for one second. The red lines indicate the trajectories over one rotation $(1 / f)$ and the grey lines show the remaining trajectories. The perpendicular bars on red trajectories indicate the nanowire's configuration projected on the XY-plane, which is scaled down 120 times from its body length. The inserts are the schematics of corresponding motions at the frequencies. (c) The corresponding drag profiles from the result of (a) along the nanowire at three different rotating frequencies. The left and right images in each panel are for the body configuration when $\theta$ is maximal and zero, respectively. All simulations start with the initial condition $d_{t}=1 \mu \mathrm{m}$. The nanowire is oriented in the $\mathrm{XZ}$ plane as an initial condition. 
We further simulated the average speed of the nanowire in the $\mathrm{Y}$ direction as a function of frequency and the result is compared to the experimental results (Figure 8a). The simulated and experimental results are normalized with respect to their respective maximum value. The normalized experimental and simulated speed show the same tendency. Up to the critical frequency $\left(f_{c}\right)$, the speed of the nanowire increases linearly with frequency of a rotating magnetic field during the tumbling motion. Above the critical frequency, the speed of the nanowire still increases but with a decreasing rate, reaching the maximum velocity around 10 $\mathrm{Hz}$, which is far below than the theatrical step-out frequency $(356 \mathrm{~Hz})$. When the body rotates with a small precession angle at high frequencies, the drag force in the $\mathrm{Y}$-axis within the period decrease (Figure 7a) and this leads to a decrease in translational velocities. Figure 8b shows the corresponding trajectories of the nanowire, moving in tumbling, precession, and rolling motion at 1,5 , and $15 \mathrm{~Hz}$, respectively.

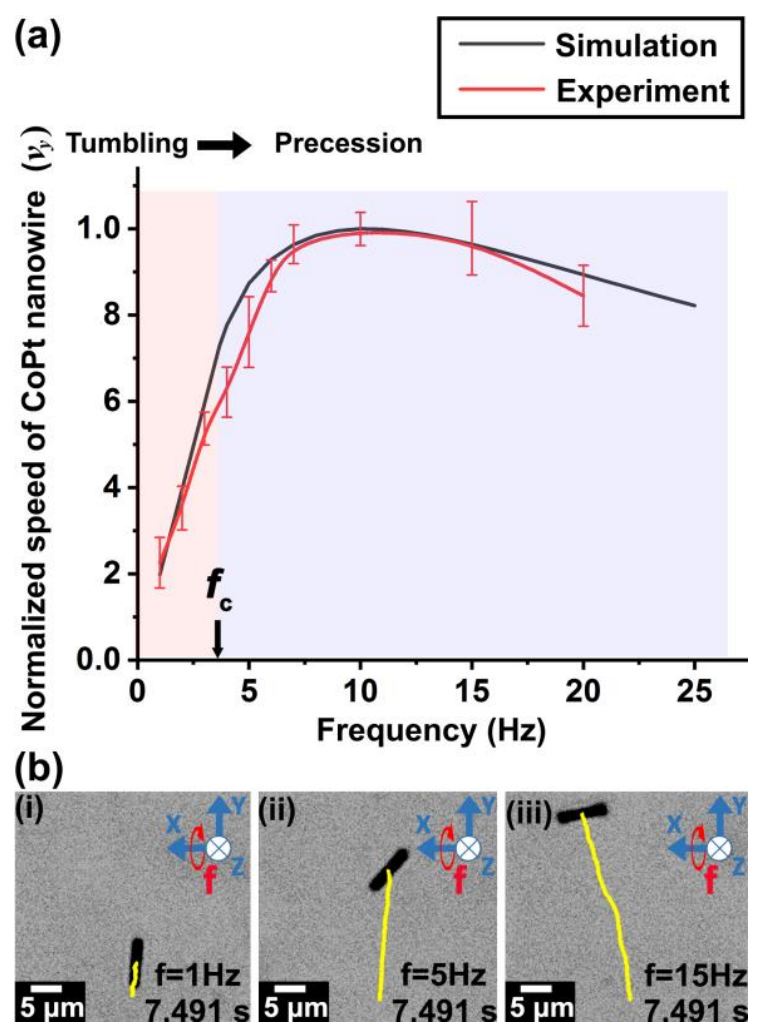

Figure 8. (a) The simulated and experimental speeds of CoPt nanowires as a function of the frequency of an applied magnetic field rotating in the YZ-plane $(B=15 \mathrm{mT})$. (b) The experimental trajectories of the CoPt nanowire at different frequencies: (i) $1 \mathrm{~Hz}$, (ii) $5 \mathrm{~Hz}$, and (iii) $15 \mathrm{~Hz}$. All trajectories were tracked over $7.491 \mathrm{sec}$. 
Additionally, we studied the influence of the distance and the magnetic moment $m$ on the speed of the nanowire. First, the effect of the distance was studied by varying the initial tip distance $(5,20,100,300$, and $500 \mathrm{~nm})$. Figure S3a shows that the speed increases when the body is located closer to the wall both in the tumbling and precession regimes. In the tumbling regime, the speed increases linearly with the applied frequency. This behavior is independent of the magnetic moment until a critical frequency $f_{c}$ at which the increased drag force triggers a precessing motion. A further study on the speed of the nanowire was conducted by varying magnetic moment $m\left(600,800,1000\right.$, and $\left.1200 \mathrm{emu} / \mathrm{cm}^{3}\right)$ at the same initial tip distance $20 \mathrm{~nm}$. We found that a higher magnetic moment $m$ allows the structure to overcome this hydrodynamic resistance, resulting in a higher peak velocity (Figure S3b). While a certain discrepancy is found between the simulated and experimental mean speeds, the simulated speeds are in good agreement with the experimental values, and at the same order of magnitude. Discrepancies originate from violating the assumption of far-field approximation made in Yang's model for small gaps from the wall, which might contribute to the enhancement of the speed in reality. Additionally, a good agreement is also found regarding the optimal frequency of maximum swimming speed, which is around $f=10 \mathrm{~Hz}$.

\section{Trapping microbeads in a vortex flow generated around a CoPt hard-magnetic}

nanowire. Structures can generate a rotating flow around their body while moving along a surface. By observing the flow, one can explain the rotating characteristic of the motion of the structure. Polystyrene (PS) beads with a diameter of 0.5 and $1 \mu \mathrm{m}$ were dispersed together with CoPt nanowires in a $60 \mathrm{wt} \%$ glycerol solution. After sedimentation of the beads onto the surface, a CoPt nanowire was magnetically manipulated to approach one of the PS beads. When a CoPt nanowire with precession motion was near a PS bead with a diameter of $0.5 \mu \mathrm{m}$, the PS bead was trapped on the tip of the CoPt nanowire and transported within the 
time recorded (Figure 8a, Movie S4). This indicates that there is a vortex formation at the tip of the nanowire, as previously reported for other structures moving in precession motion. ${ }^{19,27}$ In a similar way, rolling motion of CoPt nanowire also ensured the cargo transport of a PS bead $(1 \mu \mathrm{m})$. Figure $8 \mathrm{~b}$ shows a bead trapped at the center of the nanowire. This result is also observed in other types of structures translating in rolling motion close to the wall. ${ }^{18}$ More precisely, the bead was initially trapped at the center of nanowire's long axis and then moved to the tip of the nanowire (Movie S5). This suggests that the vortex is formed around the CoPt nanowire, but its strength increases towards the tip due to a gradual increase in drag torque. Cargo transport by CoPt nanowires using tumbling motion is not included in this letter since it has been largely reported in the previous study of Zhang, Nelson and coworkers. $^{3}$

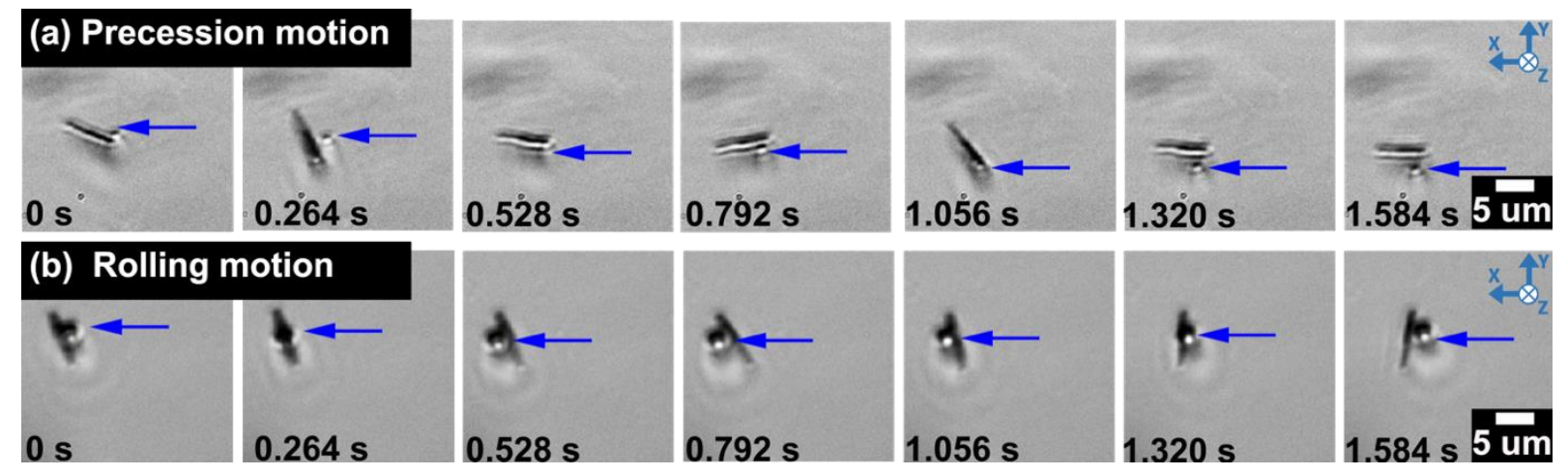

Figure 9. Particle trapping using vortexes, formed around a CoPt nanowire. PS beads, trapped by a CoPt nanowire moving with (a) precession (b) rolling motion. The blue arrows indicate the position of the trapped bead. The size of bead trapped are (a) $0.5 \mu \mathrm{m}$ and (b) $1 \mu \mathrm{m}$. 


\section{CONCLUSIONS}

In conclusion, we reported on the manipulation of pre-magnetized hard-magnetic CoPt nanowires synthesized by means of template-assisted electrodeposition. The magnetic micromanipulation experiments showed that $\mathrm{CoPt}$ nanowires are magnetized with about $25^{\circ}$ offset direction with respect to the nanowire's short axis $\left(\theta_{m} \sim 25^{\circ}\right)$. The nanowires propulsion mechanisms of tumbling, precession, and rolling were selected depending on the rotational frequency of an applied magnetic field. We also introduced theoretical model of the dynamics of the nanowires and coupled them with the boundary effect in order to predict its translational speed. The theoretical results showed that the speed is in the same order of magnitude of the experimental data and also captured its trend and existence of the optimal frequency achieving the maximal speed. Finally, we found that vortices are formed near the tip and the center of the nanowire, while presenting precession and rolling motion, respectively. This observation confirms that propulsion is primarily achieved by the strong magnetic torque, capable of overcoming drag forces. Such vortices can be used for cargo transportation tasks in which a selectable set of locomotion mechanisms provides advantages.

\section{EXPERIMENTAL METHODS}

The synthesis of CoPt nanowires. CoPt nanowires were electrochemically grown in an anodic aluminum oxide (AAO, Whatman $25 \mathrm{~mm}, 200 \mathrm{~nm}$ ) porous membrane. The filtration side of an AAO membrane was first coated with a Au layer of $200 \mathrm{~nm}$ thickness. The AAO membrane was mounted to a holder as the Au deposited face serves as a working electrode. Electrodeposition was performed in CoPt electrolyte, composed of $0.01 \mathrm{M}$ Diamminedinitrito platinum $\left(\mathrm{Pt}\left(\mathrm{NH}_{3}\right)_{2}\left(\mathrm{NO}_{2}\right)_{2}\right), 0.15 \mathrm{M}$ Cobalt sulfamate $\left(\mathrm{Co}\left(\mathrm{NH}_{2}\right)_{2}\left(\mathrm{SO}_{3}\right)_{2}\right), 0.1 \mathrm{M}$ Ammonium citrate $\left.\left(\mathrm{NH}_{4}\right)_{2} \mathrm{C}_{6} \mathrm{H}_{6} \mathrm{O}_{7}\right), 0.1 \mathrm{M}$ sodium hypophosphite $\left(\mathrm{NaPH}_{2} \mathrm{O}_{2}\right), 0.1 \mathrm{M}$ sulfamic acid $\left(\mathrm{NH}_{2} \mathrm{SO}_{3} \mathrm{H}\right), 0.1 \mathrm{M}$ glycine $\left(\mathrm{NH}_{2} \mathrm{CH}_{2} \mathrm{COOH}\right)$, and $0.005 \mathrm{M}$ saccharin 
$\left(\mathrm{C}_{7} \mathrm{H}_{5} \mathrm{NO}_{3} \mathrm{~S}\right)$. CoPt nanowires were galvanostatically deposited at $-30 \mathrm{~mA} \mathrm{~cm}{ }^{-2}$ current density at $50{ }^{\circ} \mathrm{C}$. During the deposition, Pt was used as a counter electrode. The CoPt nanowires were released by wet etching the AAO membrane in $5 \mathrm{M} \mathrm{NaOH}$ solution for 15 min. The CoPt nanowires were cleaned three times in DI water.

Pre-magnetization of CoPt nanowires. Prior to pre-magnetization, the suspension of CoPt nanowires was highly diluted with DI water. The nanowires were dropped and dried on a glass substrate, and a strong lesion was applied to the nanowires. The CoPt nanowires lying on the substrate were magnetized in such a way that the magnetization direction was perpendicular to the nanowire's long axis. Pre-magnetization was performed in a vibrating sample magnetometer (VSM) by applying $20 \mathrm{kOe.}$

Scanning electron microscopy (SEM) and energy-dispersive X-ray spectroscopy (EDX). A specimen was prepared by dispersing the solution of CoPt nanowires on a cleaned Si wafer. After the solution was completely dried, images were obtained with an ULTRA 55 plus SEM (Carl Zeiss AG, Germany).

X-ray diffraction (XRD). An XRD specimen was prepared by dispersing and drying the solution of CoPt nanowires on a glass substrate. The XRD measurement was conducted on a Bruker D8 Advance X-ray diffractometer, equipped with a $\mathrm{Cu}$ target with the wavelength of $1.542 \AA$.

Vibrating sample magnetometer (VSM). Magnetic properties of CoPt nanowires embedded in AAOs were measured using VSM (MicroSense EZ9).

Manipulation of CoPt nanowires. The magnetic manipulation of CoPt nanowires was conducted using an electromagnetic system (Nanomag Co, Magrobotix), consisting of four pairs of electromagnets arranged around a workspace. This system was mounted on an inverted optical microscope (Olympus IX) to monitor the motion. The motion was recorded at $30 \mathrm{fps}$, using a charge coupled device (CCD, Basler SCA 1400). 


\section{ASSOCIATED CONTENT \\ Supporting Information}

The Supporting Information is available free of charge on the ACS Publications website.

Figure S1, S2, and Table S1, S2 (PDF).

Movie S1. The motion of CoNi nanowire (control sample) under a xy-planar rotating magnetic field (AVI).

Movie S2. The motion of CoPt nanowire under a xy-planar rotating magnetic field (AVI) (AVI).

Movie S3. Motion transformation of CoPt nanowire as increasing the frequency of a xzplanar rotating magnetic field (AVI).

Movie S4. A vortex formation around the tip of CoPt nanowire moving with precession motion (AVI).

Movie S5. A vortex formation at the center of CoPt nanowire moving with rolling motion (AVI).

This material is available free of charge via the Internet at http://pubs.acs.org.

\section{AUTHOR INFORMATION}

\section{Corresponding Author}

*E-mail: vidalp@ethz.ch

E-mail: bnelson@ethz.ch

E-mail: izi@me.technion.ac.il

\section{Author Contributions}

B.J. and A.H. contributed equally to this work. All the authors contributed to the experiments and preparation of the manuscript. 


\section{Notes}

The authors declare no competing financial interest.

\section{ACKNOWLEDGEMENTS}

S.P. acknowledges financial support by the European Research Council Starting Grant "Magnetoelectric Chemonanorobotics for Chemical and Biomedical Applications (ELECTROCHEMBOTS)," by the ERC grant agreement n. 336456. B.J.N. acknowledges financial support by the Korea Evaluation Institute of Industrial Technology (KEIT) funded by the Ministry of Trade, Industry, and Energy (MOTIE) (no. 10052980). C.A. acknowledges financial support by the Marie Skłodowska-Curie Innovative Training Network (H2020MSCA-ITN-2014) under grant agreement 642642 (SELECTA). J.S. acknowledges partial financial support from the project 2017-SGR-292 from the Generalitat de Catalunya. E.P. acknowledges the Ramon y Cajal (RYC-2012-10839) fellowship from MINECO. Y.O. acknowledges support of the Israel Science Foundation (ISF) under grant no. 567/14, and his visit at ETH Zürich in May 2018 as part of sabbatical leave.

Received: ((will be filled in by the editorial staff))

Revised: ((will be filled in by the editorial staff)) Published online: ((will be filled in by the editorial staff))

\section{REFERENCES}

(1). Darnton, N. C.; Turner, L.; Rojevsky, S.; Berg, H. C., On torque and tumbling in swimming Escherichia coli. J. Bacteriol. 2007, 189 (5), 1756-64.

(2). Jang, B.; Gutman, E.; Stucki, N.; Seitz, B. F.; Wendel-Garcia, P. D.; Newton, T.; Pokki, J.; Ergeneman, O.; Pane, S.; Or, Y.; Nelson, B. J., Undulatory Locomotion of Magnetic Multilink Nanoswimmers. Nano Lett. 2015, 15 (7), 4829-33.

(3). $\quad$ Zhang, L.; Petit, T.; Lu, Y.; Kratochvil, B. E.; Peyer, K. E.; Pei, R.; Lou, J.; Nelson, B. J., Controlled propulsion and cargo transport of rotating nickel nanowires near a patterned solid surface. ACS Nano 2010, 4 (10), 6228-34.

(4). Gao, W.; Sattayasamitsathit, S.; Manesh, K. M.; Weihs, D.; Wang, J., Magnetically powered flexible metal nanowire motors. J. Am. Chem. Soc. 2010, 132 (41), 14403-5.

(5). Ghosh, A.; Fischer, P., Controlled propulsion of artificial magnetic nanostructured propellers. Nano Lett. 2009, 9 (6), 2243-5. 
(6). Chen, X.-Z.; Hoop, M.; Mushtaq, F.; Siringil, E.; Hu, C.; Nelson, B. J.; Pané, S., Recent developments in magnetically driven micro- and nanorobots. Applied Materials Today 2017, 9, 37-48.

(7). Chen, X.-Z.; Jang, B.; Ahmed, D.; Hu, C.; De Marco, C.; Hoop, M.; Mushtaq, F.; Nelson, B. J.; Pane, S., Small-Scale Machines Driven by External Power Sources. Advanced Materials 2018, 30 (15), 1705061.

(8). Hoop, M.; Mushtaq, F.; Hurter, C.; Chen, X.-Z.; Nelson, B. J.; Pané, S., A smart multifunctional drug delivery nanoplatform for targeting cancer cells. Nanoscale 2016, 8 (25), 12723-12728.

(9). Shields, C. W.; Velev, O. D., The Evolution of Active Particles: Toward Externally Powered Self-Propelling and Self-Reconfiguring Particle Systems. Chem 2017, 3 (4), 539-559.

(10). Ghosh, A.; Mandal, P.; Karmakar, S.; Ghosh, A., Analytical theory and stability analysis of an elongated nanoscale object under external torque. Phys. Chem. Chem. Phys. 2013, 15 (26), 10817-23.

(11). Morozov, K. I.; Leshansky, A. M., The chiral magnetic nanomotors. Nanoscale 2014, 6 (3), 1580-8.

(12). Peyer, K. E.; Zhang, L.; Nelson, B. J., Bio-inspired magnetic swimming microrobots for biomedical applications. Nanoscale 2013, 5 (4), 1259-72.

(13). Chen, X.-Z.; Hoop, M.; Shamsudhin, N.; Huang, T.; Ozkale, B.; Li, Q.; Siringil, E.; Mushtaq, F.; Di Tizio, L.; Nelson, B. J.; Pané, S., Hybrid Magnetoelectric Nanowires for Nanorobotic Applications: Fabrication, Magnetoelectric Coupling, and Magnetically Assisted In Vitro Targeted Drug Delivery. Advanced Materials 2017, 29 (8), 1605458. (14). Chen, X.-Z.; Shamsudhin, N.; Hoop, M.; Pieters, R.; Siringil, E.; Sakar, M. S.; Nelson, B. J.; Pané, S., Magnetoelectric Micromachines with Wirelessly Controlled Navigation and Functionality. Materials Horizons 2016, 3 (2), 113-118.

(15). Morimoto, H.; Ukai, T.; Nagaoka, Y.; Grobert, N.; Maekawa, T., Tumbling motion of magnetic particles on a magnetic substrate induced by a rotational magnetic field. Phys.

Rev. E Stat. Nonlin. Soft Matter Phys. 2008, 78 (2 Pt 1), 021403.

(16). Tierno, P.; Golestanian, R.; Pagonabarraga, I.; Sagues, F., Controlled swimming in confined fluids of magnetically actuated colloidal rotors. Phys. Rev. Lett. 2008, 101 (21), 218304.

(17). Sing, C. E.; Schmid, L.; Schneider, M. F.; Franke, T.; Alexander-Katz, A., Controlled surface-induced flows from the motion of self-assembled colloidal walkers. Proc. Natl. Acad. Sci. U.S.A. 2010, 107 (2), 535-40.

(18). Tung, H.-W.; Peyer, K. E.; Sargent, D. F.; Nelson, B. J., Noncontact manipulation using a transversely magnetized rolling robot. Appl. Phys. Lett. 2013, 103 (11), 114101. (19). Mair, L. O.; Evans, B. A.; Nacev, A.; Stepanov, P. Y.; Hilaman, R.; Chowdhury, S.; Jafari, S.; Wang, W.; Shapiro, B.; Weinberg, I. N., Magnetic microkayaks: propulsion of microrods precessing near a surface by kilohertz frequency, rotating magnetic fields. Nanoscale 2017, 9 (10), 3375-3381.

(20). Jiang, G.-L.; Guu, Y.-H.; Lu, C.-N.; Li, P.-K.; Shen, H.-M.; Lee, L.-S.; Yeh, J. A.; Hou, M. T.-K., Development of rolling magnetic microrobots. J. Micromech. Microeng. 2010, 20 (8), 085042.

(21). Hou, M. T.; Shen, H.-M.; Jiang, G.-L.; Lu, C.-N.; Hsu, I. J.; Yeh, J. A., A rolling locomotion method for untethered magnetic microrobots. Appl. Phys. Lett. 2010, 96 (2), 024102.

(22). Mahoney, A. W.; Abbott, J. J., Managing magnetic force applied to a magnetic device by a rotating dipole field. Appl. Phys. Lett. 2011, 99 (13), 134103. 
(23). Fomin, V. M.; Smith, E. J.; Makarov, D.; Sanchez, S.; Schmidt, O. G., Dynamics of radial-magnetized microhelix coils. Phys. Rev. B 2011, 84 (17), 174303.

(24). Ghosh, A.; Paria, D.; Singh, H. J.; Venugopalan, P. L.; Ghosh, A., Dynamical configurations and bistability of helical nanostructures under external torque. J. Appl. Crystallogr. 2012, 86 (3 Pt 1), 031401.

(25). Smith, E. J.; Makarov, D.; Sanchez, S.; Fomin, V. M.; Schmidt, O. G., Magnetic microhelix coil structures. Phys. Rev. Lett. 2011, 107 (9), 097204.

(26). Barbot, A.; Decanini, D.; Hwang, G., On-chip Microfluidic Multimodal Swimmer toward 3D Navigation. Sci. Rep. 2016, 6, 19041.

(27). Zhou, Q.; Petit, T.; Choi, H.; Nelson, B. J.; Zhang, L., Dumbbell Fluidic Tweezers for Dynamical Trapping and Selective Transport of Microobjects. Adv. Funct. Mater. 2017, 27 (1), 1604571.

(28). Lutterotti, L.; Scardi, P., Simultaneous structure and size-strain refinement by the Rietveld method. J. Appl. Crystallogr. 1990, 23 (4), 246-252.

(29). Kummer, M. P.; Abbott, J. J.; Kratochvil, B. E.; Borer, R.; Sengul, A.; Nelson, B. J., OctoMag: An Electromagnetic System for 5-DOF Wireless Micromanipulation. IEEE Trans. Robot. 2010, 26 (6), 1006-1017.

(30). Jang, B.; Pellicer, E.; Guerrero, M.; Chen, X.; Choi, H.; Nelson, B. J.; Sort, J.; Pane, S., Fabrication of segmented $\mathrm{Au} / \mathrm{Co} / \mathrm{Au}$ nanowires: insights in the quality of $\mathrm{Co} / \mathrm{Au}$ junctions. ACS Appl. Mater. Interfaces 2014, 6 (16), 14583-9.

(31). Zhang, J.; Agramunt-Puig, S.; Del-Valle, N.; Navau, C.; Baro, M. D.; Estrade, S.; Peiro, F.; Pane, S.; Nelson, B. J.; Sanchez, A.; Nogues, J.; Pellicer, E.; Sort, J., Tailoring Staircaselike Hysteresis Loops in Electrodeposited Trisegmented Magnetic Nanowires: a Strategy toward Minimization of Interwire Interactions. ACS Appl. Mater. Interfaces 2016, 8 (6), 4109-17.

(32). Kim, K.; Xu, X.; Guo, J.; Fan, D. L., Ultrahigh-speed rotating nanoelectromechanical system devices assembled from nanoscale building blocks. Nat. Commun. 2014, 5, 3632.

(33). Keshoju, K.; Xing, H.; Sun, L., Magnetic field driven nanowire rotation in suspension. Appl. Phys. Lett. 2007, 91 (12), 123114.

(34). Driscoll, M.; Delmotte, B.; Youssef, M.; Sacanna, S.; Donev, A.; Chaikin, P., Unstable fronts and motile structures formed by microrollers. Nat. Phys. 2016, 13, 375.

(35). Yang, S.-M.; Leal, L. G., Particle motion in Stokes flow near a plane fluid-fluid interface. Part 1. Slender body in a quiescent fluid. J. Fluid Mech. 2006, 136, 393-421.

\section{Table of Contents artwork}




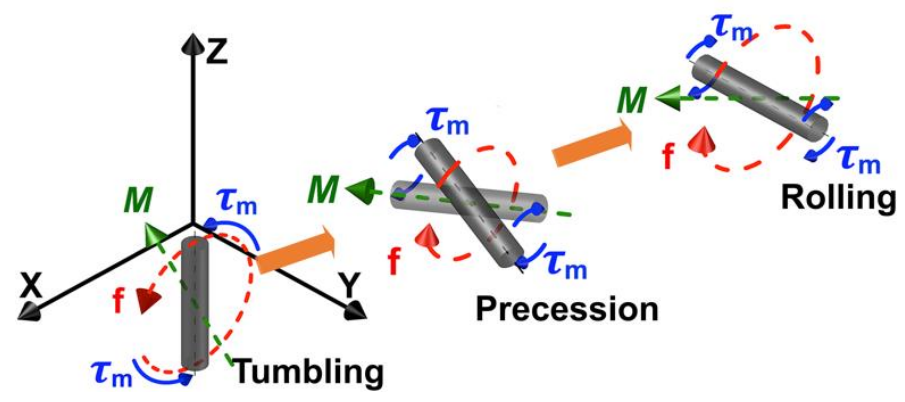

